\title{
Determination of the Residues of the Insecticide Lambda-cyhalothrin After Heat Treatment Using HPLC and Biological Evaluation
}

\author{
Laith Osama Mohammad Amin Al-Ramadany ${ }^{*}$, Nabil Mustafa Taha Al Mallah² \\ ${ }^{1 *, 2}$ Faculty of Agriculture and Forestry, Department of Plant Protection, University of Mosul, Mosul, \\ Iraq \\ E-mail: ${ }^{1 *}$ lith_81@yahoo.com, ${ }^{2}$ dr.almallah@uomosul.edu.iq
}

(Received June 10, 2021; Accepted July 10, 2021; Available online September 01, 2021)

DOI: 10.33899/edusj.2021.130469.1166, (c) 2021, College of Education for Pure Science, University of Mosul.

This is an open access article under the CC BY 4.0 license (http://creativecommons.org/licenses/by/4.0/).

\begin{abstract}
The present study was conducted with the aim of evaluating the effect of using three temperatures of 30,40 , and $50{ }^{\circ} \mathrm{C}$ and five exposure periods of $6,12,24,48$ and 96 hours/degree in reducing Lambda-cyhalothrin residues in water under laboratory conditions and measuring these residues using HPLC as well as Biological evaluation of these residues before and after thermal treatments. The HPLC readings of the residues of this pesticide showed that the highest general average of the degradation rate was at a concentration of $30 \mathrm{ppm} /$ active substance $(93.47 \%)$ and at a temperature of $30{ }^{\circ} \mathrm{C}(94.79)$ and for an exposure period of 48 hours $(89.80 \%)$. and that the percentage of deterioration in the effectiveness of the pesticide against adults of the insect T.confusum ranged between 96.72 and $99.22 \%$, and the highest of these percentages was at a concentration of $10 \mathrm{ppm}(99.22 \%)$ and at a temperature of $40^{\circ} \mathrm{C}(98.33 \%)$ and for the two exposure periods 24 and 48 hours $(98.29 \%)$. This may indicate the sensitivity of this pesticide to the temperatures used, which was apparently high in its initial limit, while the highest rate of degradation was $89.80 \%$ at the 48 -hour period, which differed significantly from the rest of the other averages, which amounted to $82.2,85.26,89.35$ and $86.95 \%$ for exposure periods of $6,12,24$ and 96 hours, respectively.
\end{abstract}

Keywords: Lambda-cyhalothrin, temperature, HPLC

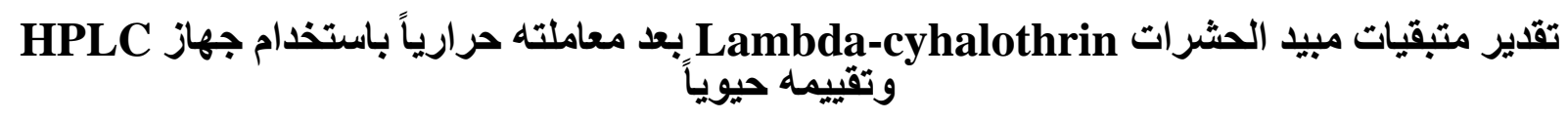

$$
\begin{aligned}
& \text { ليث أسامة محمدأمين الرمضاني *1، نبيل مصطفى طه الملاح² } \\
& \text { 1*2، كلية الزر اعة و الغابات، قسم وقاية النبات، جامعة الموصل، الموصل، العراق }
\end{aligned}
$$

الخلاصة

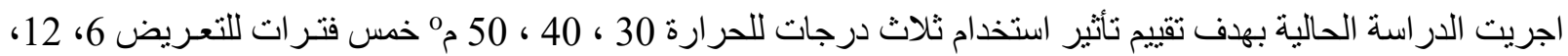

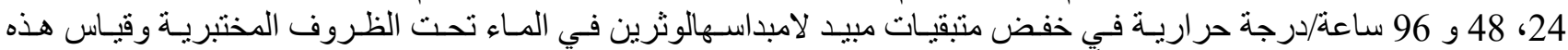

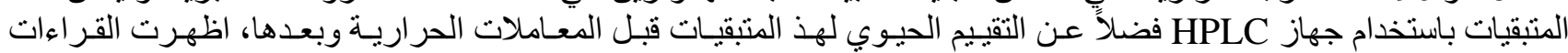

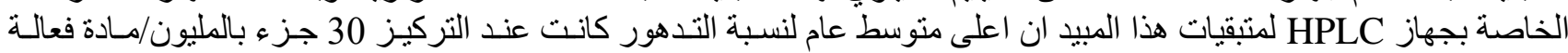

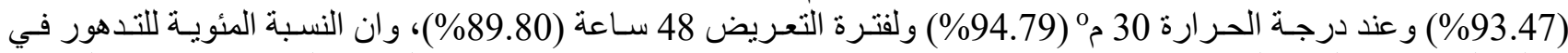

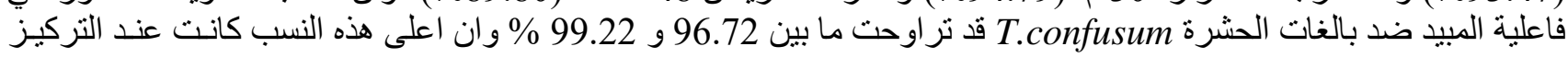




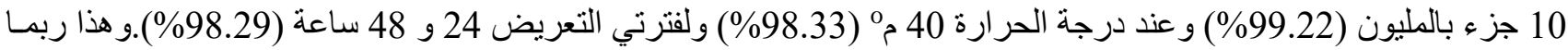

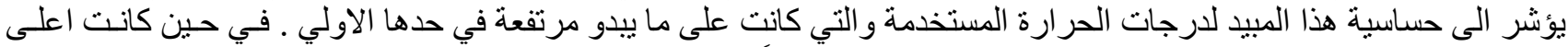

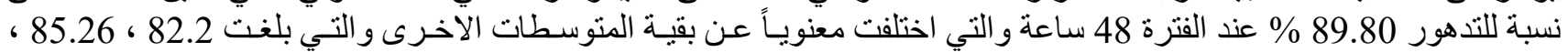

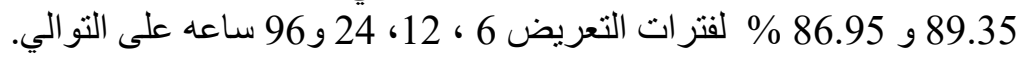

الكلمات المفتاحية: لامبداسهالوثرين، درجة الحرارة، HPLC

\section{المقدمـة}

تعد المبيدات الكيميائية اهم مكون في اي استراتيجية مقترحسة لإدارة الآفات لذلك فقد حظيت بأهمية خاصـة في جدول

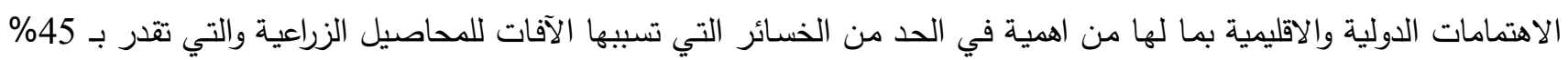
من الانتاج الزراعي العالمي، منظمه الأغذية والزراعة [1] حيث تلعب دورا رئيسياً في الحفاظ على انتاجيه زراعيه عالية في مدخلاتها

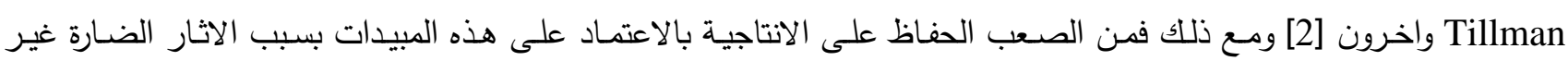

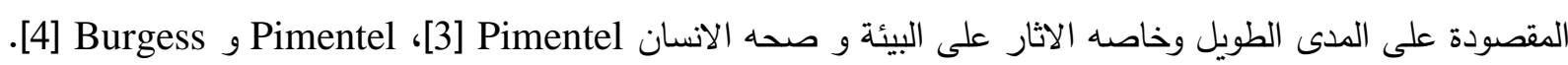
ان متبقيات المبيدات موجوده في جميع النظم البيئية الزراعية ولكن الخطر الحقيقي على صحه الانسـان هو من خـلال

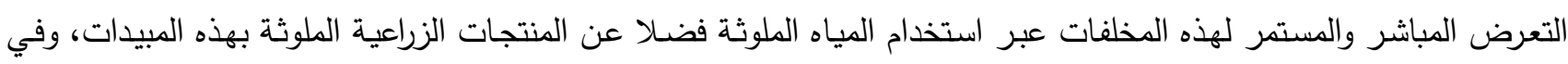

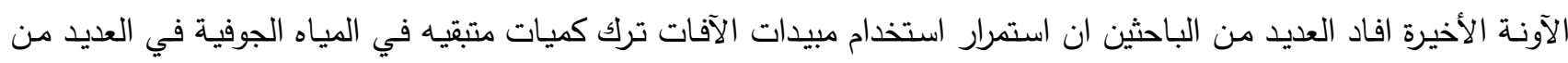

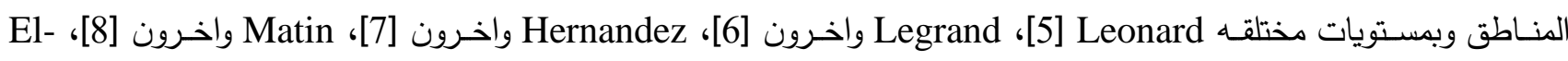

Kabbany وان تلوث مصادر المياه الجوفية بهذه المبيدات تسبب في زيـاده القلق البيئي وخاصة في البلدان التي تثكل فيها المياه

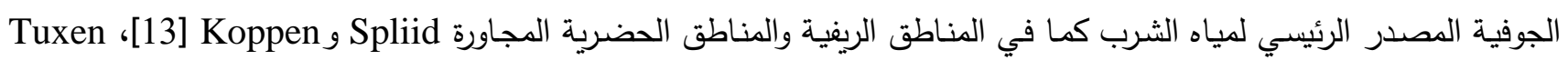
واخرون [14]، Papadopoulo-Mourkidou واخرون [15] خاصـه اذا علمنـا ان هناك حوالي مليار شخص ليس ليس لديهز مياه شرب صحيه وان هناك اكثر من ستة ملايين شخص (بينهم مليون طفل) يموتون بسبب السرطان والملاريا التي تسببها مياه الشرب .[16]vara

مبيد م: Lambda-Cyhalothrin من المبيدات البايرثرويديه التي تم تسجيلها في عام 1988 من قبل وكاله حمايه البيئة الأمريكية EPA [17 على انه نوع جديد من مبيدات الحشرات المصنعة وامتلاكها لقدرات عالية تعود لفاعليتها العالية بتركيزات


من سوق المبيدات الحشرية العالمية ولأكثر من3500 تركيبه مسجله تستخدم على نطاق واسع في الزراعة والمناطق السكنية والصحة

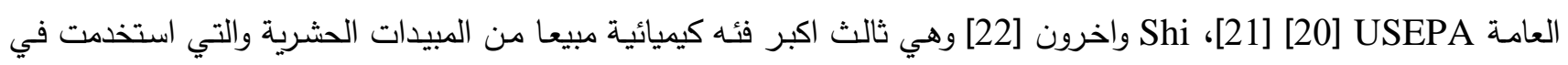
علاج 320 مليون هكتار Dousset و Dickman [23 وان تخليق هذه الفئه ومنها مبيد لامبداسهالوثرين هو نتيجة عمل الباحثين [24 Peterson و Schleier لإيجاد صيغه اكثر مقاومة للتحلل الضونئي

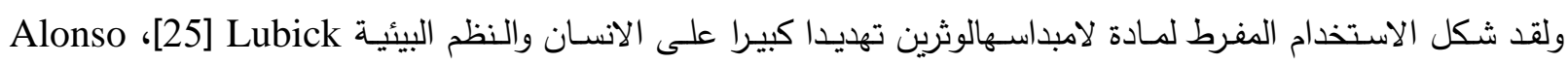
واخرون [26] وان سمية لامبداسهالوثرين على الكائنات المائية عالية عندما تصل الى الماء و تزداد عند زياده التراكيز فيه بالرغم

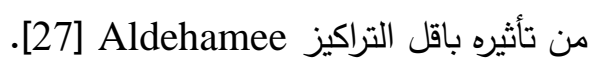

تثير الدراسات الى ان درجه الحرارة هي عامل فيزيائي مهم يؤثر على سميه المبيدات الحشرية وعلى اداء ونشاط المركبات

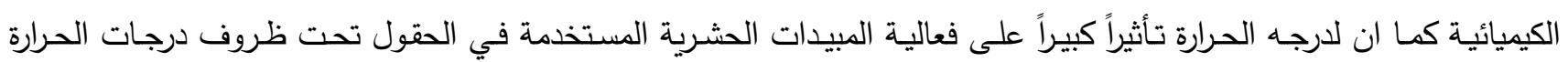


المختلفة Gordon، [28] يعتمد تأثير درجـه الحرارة على المبيدات الكيميائية بعوامل عديده منها التركيب الكيميائي للمبيد نفسـه

[29] واخرون Sparkas

ذكر Bloomfield واخرون [30] دراسة تأثير تغير درجة حرارة على سلوك ومصير مبيدات المياه السطحية والجوفية من

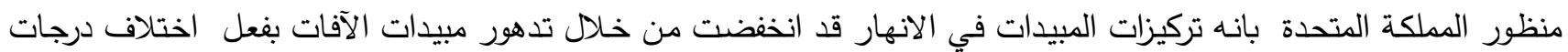
الحرارة.

ان احد العوامل التي تؤثر على معدل تحلل المواد الكيميائية بما في ذلك منتجات وقاية النبات هي درجة الحرارة كما وان لكل مبيد من المبيدات له معامل معدل تحلل كدالة لارجه الحرارة وفقا لمعادلة ارهينيوس Arrhenius حيث يمكن من خلالها

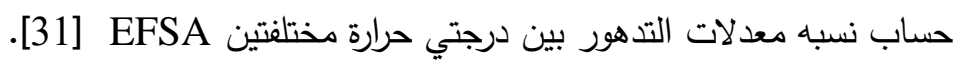
وتعد تقنية HPLC شكل من كروماتوغرافيا العمود المستخدمة بشكل عام في الكيمياء الحيوية والتحليلية ومن اكثر التقنيات



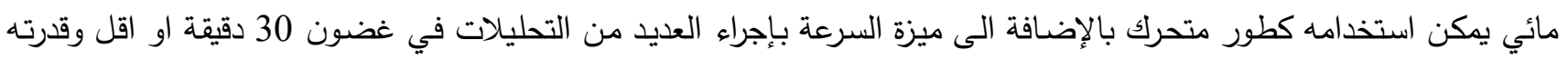

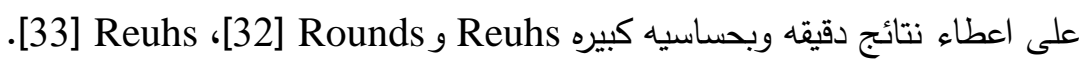
ان دراسات عديده في هذا المجال اشارت الى استخدام هذه التقنية وبنجاح في تقدير متبقيات العديد من مبيدات الحشرات



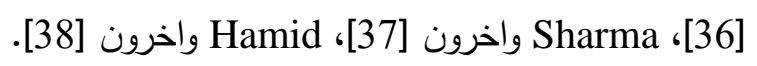
وبالنظر لمحدودية الدراسات السابقة من حيث الطرائق المستخدمة وكذلك على مبيدات بعينها فضلا عن افتقارها لتجارب التقييم الحيوي التي تعد ضرورية لمعرفه التأثير السمي لهذه المتبقيات قبل وبعد المعالجة فان الدراسة الحالية تهدف الى تقييم تأثير

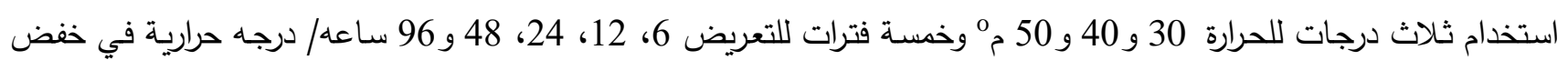
متبقيات هذا المبيد في الماء تحت الظروف المختبريـة فضلا عن التقييم الحيوي لهذه المتبقيات قبل وبعد المعاملات الحراريـة لان



المواد وطرائق العمل

اجريت التجارب في مختبر الحشرات التابع لقسم وقاية النبات والمختبر المركزي لكلية الزراعة والغابات / جامعة الموصل خلال العام 2019 المواد والأجهزة المستعطلة في الدراسة : 1

Tribolium confusum Duval Confused flour beetle تم الحصول على حشرة خنفساء الطحين المتثابهة (Coleoptera: Tenebrionidae) عزل الحشرات الكاملة منها بوساطة الغربلة وتربية الحشرة على دقيق القدح (الطحين) نظيف وسليم وضع في علب بلاستيكية سعة 500 غرام نظيفة محكمة الغلق بواسطة قطع من القماش (ململ) وربطها بوساطة اربطة مطاطية ووضعها في الحاضنة على درجة

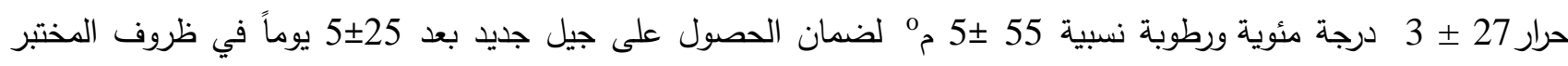
بالإضافة الى تتطيف الطحين باستمرار من جلود الانسلاخ واخذ اليرقات لعمل مزارع جديدة للحشرات الكاملة الناتجة واستخدامها في


2- المبيد كراتي (Karate 100g CS) 
مبيد حشري صاعق من مجموعة البايرثريدات (Pyrethroids) مجزز على شكل كبسولات معلقة Capsule) (CS) Suspension مواد حاملة ،الصيغة الكيميائية a-cyano-3-phenoxybenzyl3-(2-chloro-3,3,3-trifluoroprop-1-enyl)-2,2

Dimethylcyclopropanecarboxylate

WHO Class II لوقاية المزروعات بازل/ سويسرا مصنف حسب منظمة الصحة العالمية Syngenta منتاج شركة سنجنتا متوسط السمية.

- حاضنة SINDEbator كهربائية ماركة BINDER المانية الصنع الموديل BD53 درجة الحرارة من 5 درجات مئوية فوق درجة

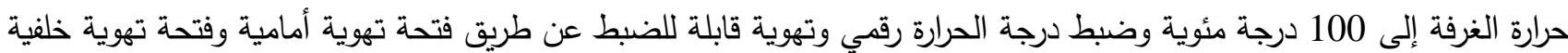
قطرها 50 مليمتر وباب زجاجي داخلي ، طاقتها الاستيعابية 115 لترًا القياسات الداخلية العرض 400 ، الارتفاع 400 ، العمق • 330

4- فرن كهربائي Oven ماركة Genlab صنع المملكة المتحدة UK الموديل MINO/40 المدى الحراري من 30- 250 درجة مئوية والتحكم بدرجة الحرارة يدوي سعته 40 لتر الارتفاع 580 ميليمتر، العرض 480 ميليمتر ، العقق 490 ميليمتر. 5- جهاز الكروماتوكرافي السائل عالي الاداء High Performance Liquid Chromatography (HPLC) Inertsil OSD-3 (C18) مواصفات العمود , LC-2010A HT اليابانية الموديل SHIMADZU CORPORATION 5رm 4.6 x 250 mm يعمل من خلال برنامجه الخاص على الحاسوب بالإضافة الى لوحة التحكم فيه. الدراسات : وشملت اولا : تجارب التقييم الحيوي للمبيدات : وتوزعت هذه التجارب على محورين :

1. التقييم الحيوي للمبيد قبل التعريض للحرارة :

ومنها يتم تحديد قيم السمية الحادة للمبيد في بالغات خنفساء الطحين المتثابهة لتتفيذ الدراسة تم اختيار أربعة تراكيز من المبيد لامبداسهالوثرين (LCY) والذي تم تحديدها استتادا الى تجارب اوليه تمهيديه أجريت


عوملت الحشرات البالغة بواقع ثلاث مكررات / تركيز وضم المكرر الواحد 10 حشرات وضعت في طبق بلت بتري حجم 9 ملم بعد

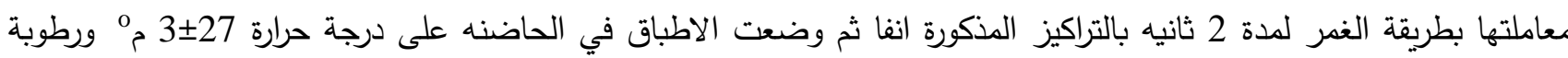

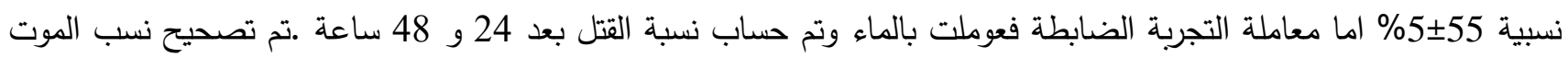
باستخدام معادلة Abbott [41]، شعبان والملاح [42] كما تم حساب قيم LC50 وحدود الثقة والميل لكل مبيد باستخدام الحاسوب

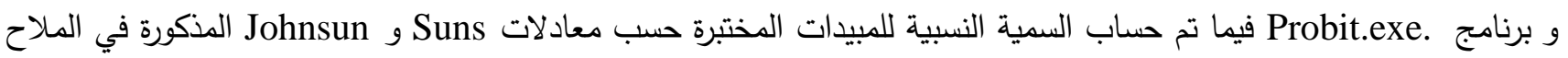
وعبدالرزاق [43]. $100 \mathrm{X}$ \% للموت في المعاملة - \% للموت في المقارنة

$$
\begin{aligned}
& 100 \text { - ل للدوت في المقارنة } \\
& \text { 2. التقييم الحيوي للمبيدات بعد التعريض للحرارة : }
\end{aligned}
$$

من اجل تحديد التأثير التثبيطي او التتشيطي للمعاملات الحرارية (درجات حراره وفترات تعريض مختلفة) في فاعلية او كفاءة




تم تحديد تركيزين/ مبيد واستتادا الى ما تم الحصول عليه من نتائج من المحور الأول وهذه التراكيز هي 10 و 30 جزء

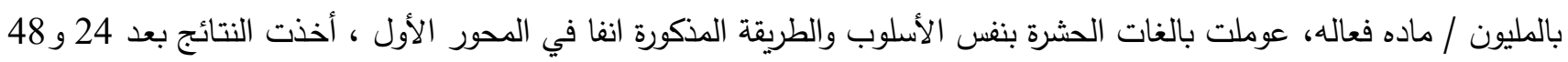

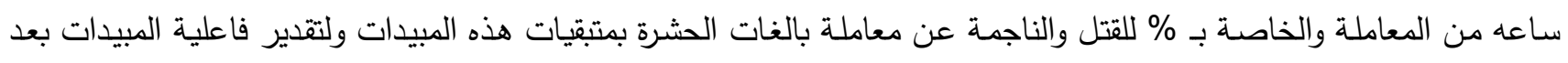

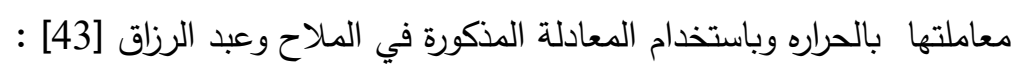

نسبة القتل قبل المعاملة الحرارية - نسبة القتل بعد المعاملة $100 \mathrm{X}$ \% للتدهور في فاعلية المبيد =

نسبة الموت قبل المعاملة (المقارنة)

ثانيا: تجارب التعريض للحرارة :

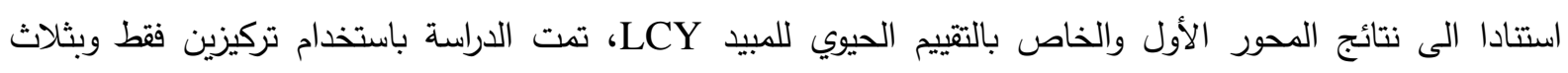

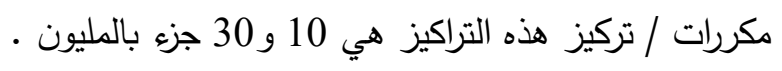

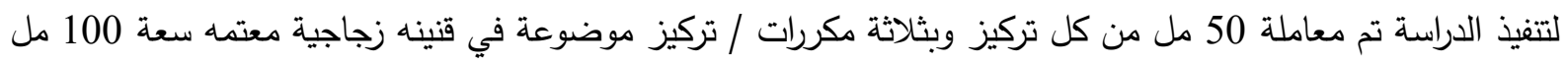
محكمة الغلق لاستبعاد احتمالية تأثير عامل الضوء في تحلل او تدهور هذه المبيدات، وضعت مكررات التجربة جميعاً في حضان

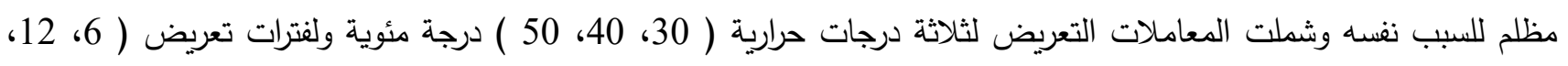
24، 48، 96 ) ساعه / درجه حرارية. بعد اكتمال تعريض العينات السابق ذكرها من المبيدات ومن اجل معرفة تدرة تأثير درجة الحرارة والتركيز وفترة التعريض لمبيد LCY فقد تم الكثف عن ذلك بطريقتين : أ- الكشف الكيميائي باستخدام جهاز ب- التقييم الحيوي لمتبقيات المبيد المعامل باسن

أ- الكشف باستخدام جهاز HPLC

بعد تحضير المحاليل القياسية للمبيد وبالتراكيز السابق ذكرها (تركيزين/ مبيد)، حلت العينات بوساطة جهاز كروماتوغرافيا

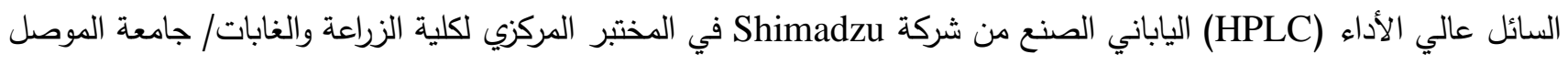
ويتكون الجهاز من طور متحرك قطبي يحتوي على مزيج من المذيبات (تختلف باختلاف المادة المطلوب الكثف عنها) والطور الثابت يتألف من العمود الذي يكون عادة من الستانلس ستيل (Stainless steel) والحاوي على جزئئات السليكا، اجري التحليل لمبيد LCY تحت ظروف عمل لجهاز HPLC





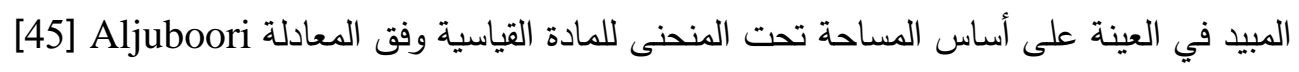
تركيز المبيد في العينة ( مايكروغرام/ مل)=تركيز المادة القياسية للمبيد xساحة منحنى المبيد المعامل مساحة منحنى المادة القياسية للمبيد 


\section{ب- التقييم الحيوي للمبيد بعد المعاملة الحرارية :} نفذت الدراسـة باستخدام بالغات خنفساء الطحين المتشـابهة Tribolium confusum ككائن اختبار حيوي لتحديد اثر

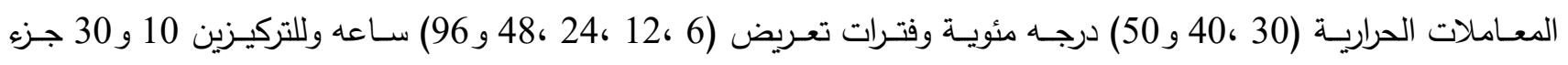
بالمليون/ماده فعاله من مبيد LCY في السمية الحادة لمتبقيات هذا المبيد. عوملت بالغات الحشرة بالمبيدات بطريقة الغمر وكما سبق ذكره في تجارب التقييم الحيوي واخذت النتائج الخاصة بـ \% للموت بعد 24 و 48 ساعه من المعاملة .

حلت البيانات وفق نظام التجارب العاملية باستخدام التصميم العشوائي الكامل C.R.D وتم استخدام اختبار دنكن متعدد

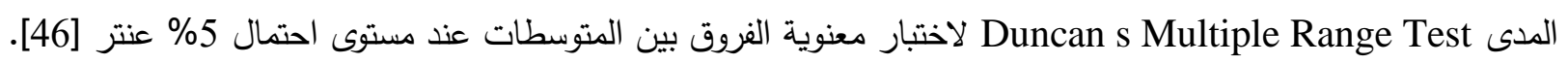

النتأسج والمناقشـة: تأثير المعاملة بالمبيد LCY) Lambda-cyhalothrin) قبل تعريضه للمعاملات الحرارية في السمية الحادة لبالغات خنفساء الطحين المتشابهة T.confusum يتبين من الجدول (1) ان مبيد LCY اظهر تأثيرا في متوسط نسبه القتل لبالغات خنفساء الطحين المتثابهة




اظهرت نتائج التحليل الاحصائي وجود فروقات معنوية في متوسط نسبه القتل تبعاً للتركيز والفترة من المعاملة وان اعلى متوسط

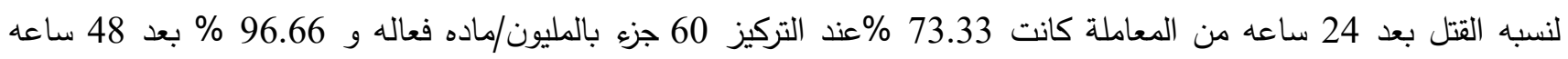
لنفس التركيز اما بالنسبة للمتوسط العام للتأثير فقد اثبت التحليل الاحصائي للتداخل بين المبيد والفترة من المعاملة وجود فرق معنوي وان اعلى متوسط عام لتأثير الفترة كان 78.33 \% للفترة 48 ساعهام .

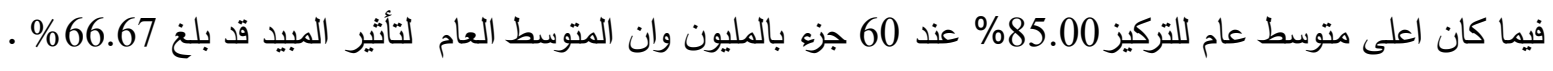

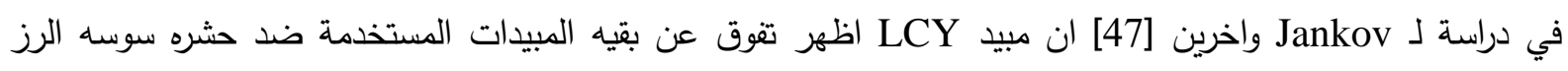
Sitophilus oryzae الخرسانية واظهر ايضا تفوقه على فترات زمنية مختلفة بعد المعاملة وصلت الى 90 يوما وعند التعريض لمده 24 ساعة.

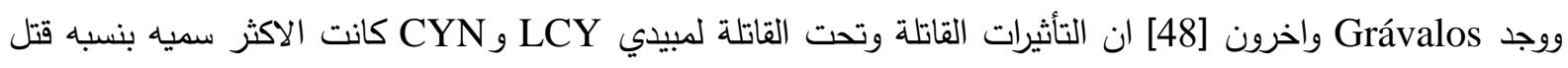

بلغت اكثر من 80\% للطور اليرقي والبالغ لمفترس Convergens Hippodamia من عائله

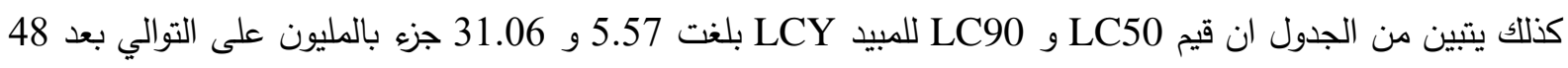

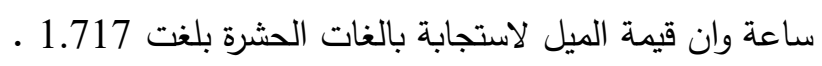
ففي دراسة لمقارنة سمية تسعة مبيدات الحشرات ضد بالغات خنفساء الطحين الصدئية T.castanum اشار الباحثان

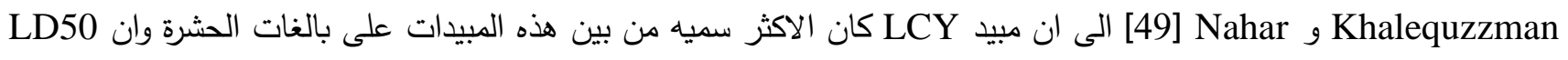



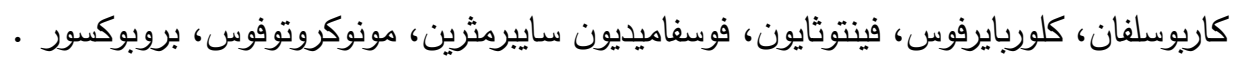


الجدول (1) نتائج التقييم الحيوي وبعض مقاييس السمية الحاده للمبيد Lambda-cyhalothrin ضد بالغات حشرة خنفساء الطحين المتشابهة

\begin{tabular}{|c|c|c|c|c|c|c|c|c|c|}
\hline \multicolumn{10}{|c|}{ T.confusum تحت الظروف المختبرية. } \\
\hline \multirow{2}{*}{$\begin{array}{c}\text { LC90 } \\
\text { ppm }\end{array}$} & \multirow{2}{*}{$\begin{array}{c}\text { LC50 } \\
\text { ppm }\end{array}$} & \multirow{2}{*}{ حدود الثقة } & \multirow{2}{*}{  } & \multirow{2}{*}{ المتام لتأثير } & \multirow{2}{*}{ متوسط التركيز } & \multicolumn{2}{|c|}{ متوبط \% القتل بعد / ساعة } & \multirow{2}{*}{  } & \multirow{2}{*}{ المبيد } \\
\hline & & & & & & 48 & 24 & & \\
\hline \multirow{4}{*}{31.06} & \multirow{4}{*}{5.57} & \multirow{4}{*}{$9.89-0.53$} & \multirow{4}{*}{1.717} & \multirow{4}{*}{66.67} & $38.33 b$ & $46.66 \mathrm{~cd}$ & $30 \mathrm{~d}$ & 5 & \multirow{4}{*}{$\begin{array}{c}\text { Lambda- } \\
\text { cyhalothrin } \\
\text { (LCY) }\end{array}$} \\
\hline & & & & & $68.33 \mathrm{a}$ & $83.33 \mathrm{ab}$ & $53.33 \mathrm{~cd}$ & 15 & \\
\hline & & & & & $75.00 \mathrm{a}$ & $86.66 \mathrm{ab}$ & $63.33 \mathrm{bc}$ & 30 & \\
\hline & & & & & $85.00 \mathrm{a}$ & $96.66 \mathrm{a}$ & $73.33 \mathrm{abc}$ & 60 & \\
\hline & & & & & & $78.33 \mathrm{a}$ & $55.55 \mathrm{~b}$ & ل للفترة &  \\
\hline
\end{tabular}

"المتوسطات ذات الاحرف غير المتثابهة في القطاع الواحد تثير الى وجود فروقات معنوية عذد مستوى احتمال 5\%.

تأثير درجات الحرارة المختلفة وفترات التعريض في النسبة المئوية لتدهور مبيد التيد

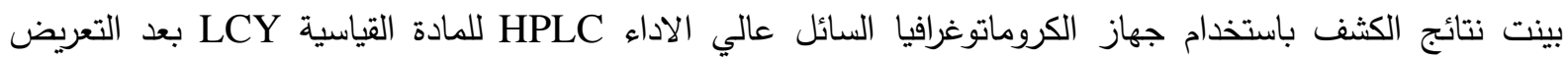
للحرارة الثكل (1) لمستحضر المبيد LCY بعد التعريض للحرارة والموضح في الجدول (2) التأثير الواضح لاستخدام درجات الحرارة


النسبة قد تراوحت ما بين 59.73 و 96.06 \% للتركيز 10 جزء بالمليون من المادة الفعالة والتي سجلت عند درجتي الحرارة 50 ،


30 جزء بالمليون ماده فعالة والتي سجلت عند درجتي الحرارة 50 و 30 م ايضا ولفترتي التعريض 6 و و24 ساعه على التوالي.



1 Det.ACh1/230nm

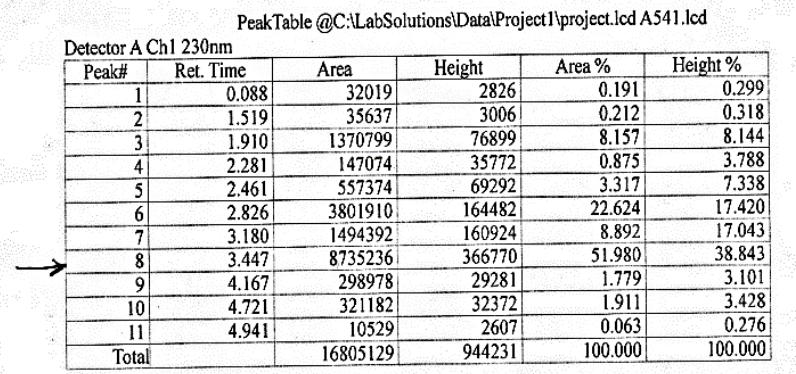

الثكل (1) مخطط المبيد الذي يُظهره جهاز HPLC والمستخدم لحساب التراكيز 
وقد اظهرت نتائج التحليل الاحصائي وجود فروقات معنوية في متوسط النسبة المئوية لتدهور مبيد LCY تبعا للتركيز و

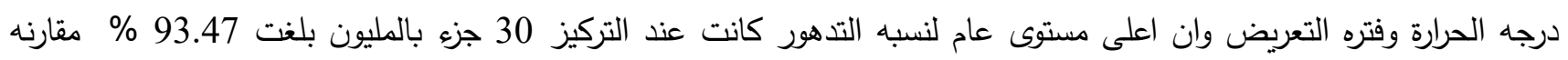

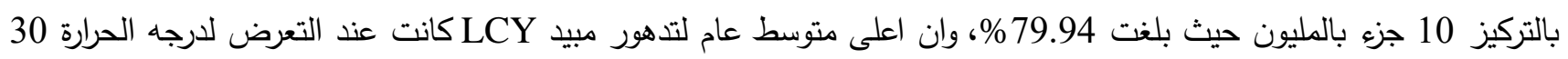





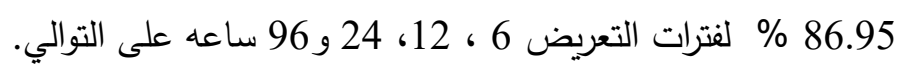

ان هذه النتائج على ما يبدو للوهلة الاولى انها قد لا تتفق مع العديد من الدراسات التي تثير الى ان تحلل بشكل عام يزداد



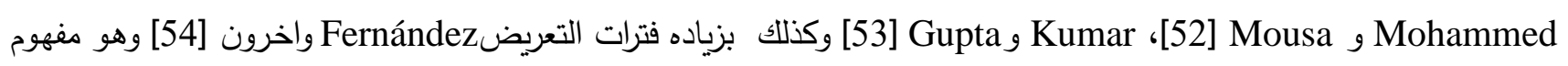
عام يحتاج الى تفصيل لتبرير وتأكيد ما تم التوصل اليه في دراستتا الحالية وذلك اذا علما ان المبيدات البايرثريدية ومنها مبيد







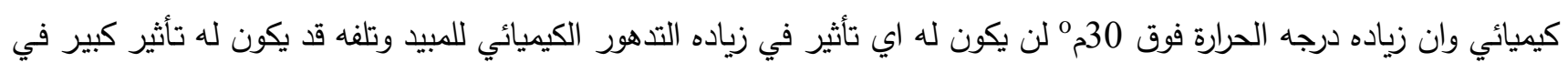



المبيد كان داخل قناني زجاجيه مغلقه ومعتمة وبالتالي فان تأثير الضوء وتطاير او تبخر المبيد كان مستبعداً عند اجراء التجربة.

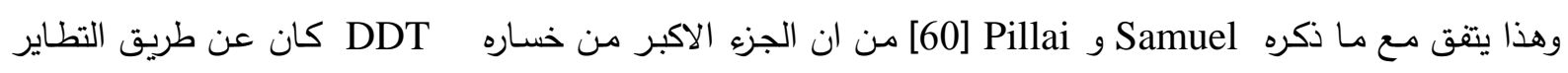

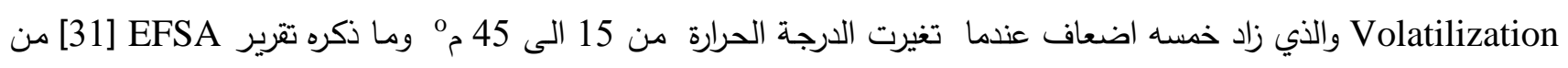

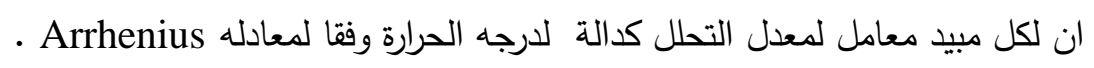
اما بالنسبة لتأثير التداخل بين الحرارة والتركيز في متوسط \% لتدهول لتهور مبيد LCY فقد اظهر التحليل الاحصائي وجود

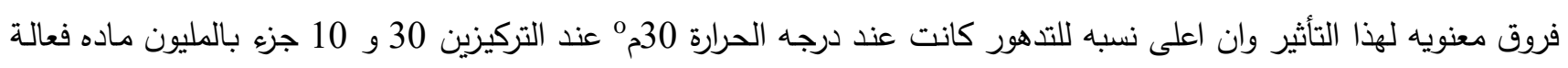


جزء بالمليون. اما بالنسبة لتأثير التداخل بين الحرارة وفتره التعريض فان نتائج الجدول (2) تشير الى ان اعلى متوسط لنسبه التدهور تم تسجيلها

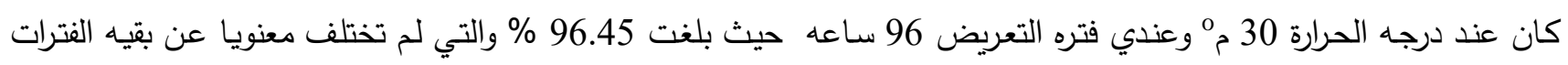

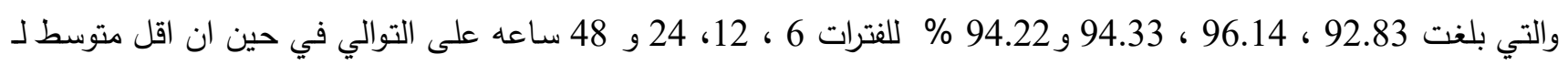
\% لتدهور المبيد قد بلغت 72.32 \% والتي سجلت عند التعريض لدرجه الحرارة 50 م للفترة 6 ساعه.

نتائج التقييم الحيوي للمبيد Lambda-cyhalothrin بعد التعريض للمعاملات الحرارية

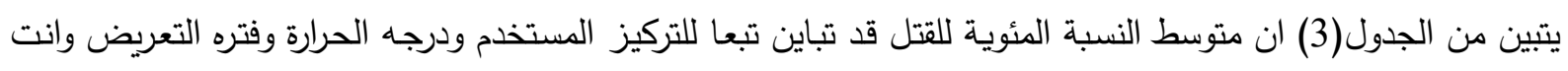

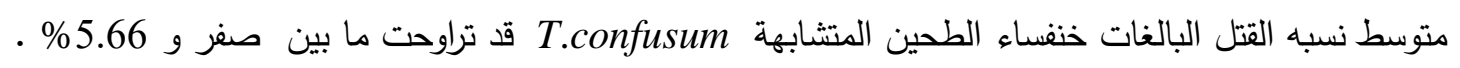

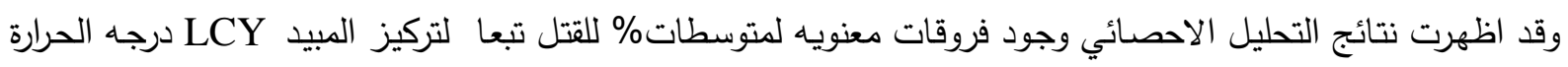

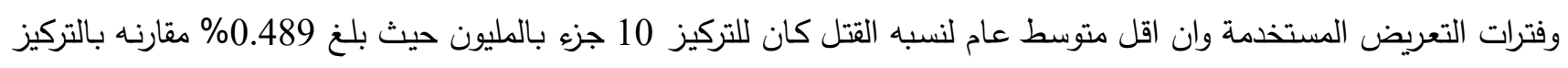

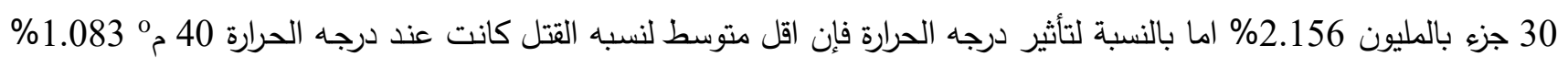




التي تم تسجيلها عند درجه الحرارة 50 م حيث بلغت 1.633\% اما بالنسبة لتأثير فترات التعريض فان اقل متوسط عام لنسبه القتل

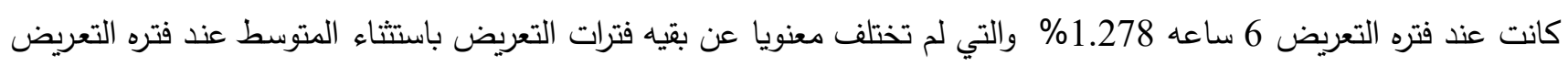
96 ساعه 1.75\% التي اختلفت معنويا عن جميع فترات التعريض الاخرى المستخدمة.






على التوالي اما بالنسبة لتأثير التداخل بين الحرارة وفتره التعريض فان اقل متوسط للقتل تم تسجيله 0.583 \% كان عند درجه الحرارة



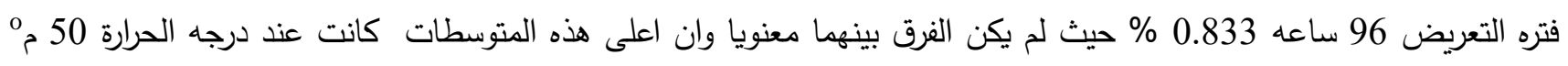
ولفتره التعريض 96 ساعه حيث بلغت 3.25\% والتي اختلفت معنويا عن جميع متوسطات \% م للقتل الاخرى.



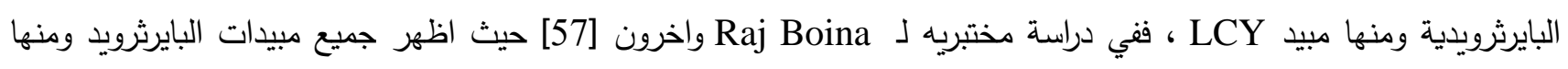

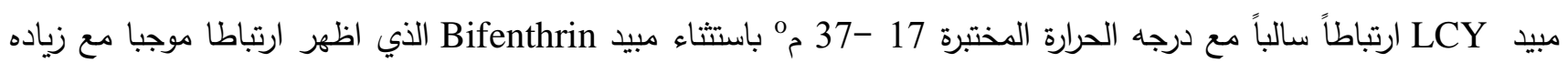



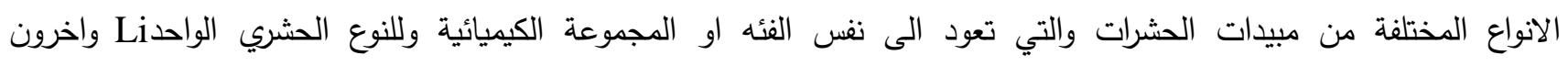

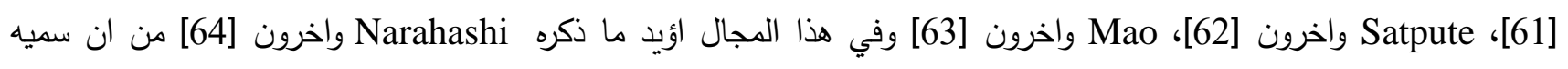
المبيدات تتأثر بدرجه كبيره بالحرارة وان الاليه المسؤولة عن ذلك تحتاج الى مزيد من الدراسات لتوضيح حقيقه او ماهيه هذه العلاقة 
Journal of Education and Science (ISSN 1812-125X), Vol: 30, No: 4, 2021 (161-176)

الجدول (2) نتائج الكثف باستخدام جهاز الكروماتوغرافيا السائل عالي الاداء HPLC لمبيد Lambda-cyhalothrin بعد معاملته حرارياً

\begin{tabular}{|c|c|c|c|c|c|c|c|c|c|c|c|}
\hline \multicolumn{3}{|c|}{ المتوسط العام لتأثير } & \multicolumn{2}{|c|}{ المتوسط لتأثير التداخل } & \multirow[b]{2}{*}{ \% تدهور المبيد } & \multirow[b]{2}{*}{$\begin{array}{c}\text { / التركيز النهائي } \\
\text { Ppm }\end{array}$} & \multirow[b]{2}{*}{ |نكستروم Aساحة المنحنى/ } & \multirow[b]{2}{*}{ |زمن الاحتباس| } & \multirow[b]{2}{*}{ فترة التعريض/ ساعة } & \multirow[b]{2}{*}{ الحرارة المستخدمة/ } & \multirow[b]{2}{*}{$\begin{array}{c}\text { / التركيز } \\
\text { ppm }\end{array}$} \\
\hline | التركيز/ & التعريض/ سترة & مُ مُارة| & التركيز & الحرارة فترة & & & & & & & \\
\hline \multirow{14}{*}{$79.94 b$} & $82.2 \mathrm{e}$ & \multirow{5}{*}{$94.97 \mathrm{a}$} & \multirow{5}{*}{$92.12 \mathrm{c}$} & $92.83 \mathrm{~d}$ & 89.51 & 1.049 & 916511 & 3.303 & 6 & \multirow{5}{*}{30} & \multirow{14}{*}{10} \\
\hline & $85.26 \mathrm{~d}$ & & & $96.14 a$ & 94.20 & 0.580 & 506871 & 3.307 & 12 & & \\
\hline & $89.35 b$ & & & $94.33 b$ & 90.43 & 0.957 & 836055 & 3.327 & 24 & & \\
\hline & $89.80 \mathrm{a}$ & & & $94.22 \mathrm{c}$ & 90.43 & 0.957 & 542412 & 3.453 & 48 & & \\
\hline & $86.95 c$ & & & $96.45 a$ & 96.06 & 0.394 & 344634 & 3.294 & 96 & & \\
\hline & & \multirow{5}{*}{$84.73 b$} & \multirow{5}{*}{$75.63 \mathrm{e}$} & $84.44 \mathrm{k}$ & 69.20 & 3.080 & 2690737 & 2.971 & 6 & \multirow{5}{*}{40} & \\
\hline & & & & $83.9 \mathrm{j}$ & 75.60 & 2.440 & 2131921 & 2.964 & 12 & & \\
\hline & & & & $84.72 \mathrm{i}$ & 77.55 & 2.245 & 1961402 & 2.825 & 24 & & \\
\hline & & & & $86.61 \mathrm{~h}$ & 76.65 & 2.335 & 2040478 & 2.858 & 48 & & \\
\hline & & & & $86.97 \mathrm{~g}$ & 79.15 & 2.085 & 1821818 & 2.831 & 96 & & \\
\hline & & \multirow{8}{*}{$80.62 \mathrm{c}$} & \multirow{4}{*}{$72.09 \mathrm{f}$} & $72.32 n$ & 59.73 & 4.027 & 3518457 & 3.065 & 6 & \multirow{4}{*}{50} & \\
\hline & & & & $75.74 \mathrm{~m}$ & 63.28 & 3.672 & 3207960 & 2.922 & 12 & & \\
\hline & & & & $89.01 \mathrm{e}$ & 87.94 & 1.206 & 1053875 & 2.924 & 24 & & \\
\hline & & & & $88.58 \mathrm{f}$ & 81.99 & 1.801 & 1573307 & 2.958 & 48 & & \\
\hline \multirow{14}{*}{$93.47 \mathrm{a}$} & & & \multirow{4}{*}{$97.46 \mathrm{a}$} & & 98.08 & 0.574 & 501949 & 3.369 & 12 & \multirow{4}{*}{30} & \multirow{14}{*}{30} \\
\hline & & & & & 98.23 & 0.529 & 462665 & 3.370 & 24 & & \\
\hline & & & & & 98.02 & 0.592 & 517741 & 3.361 & 48 & & \\
\hline & & & & & 96.84 & 0.947 & 827883 & 3.334 & 96 & & \\
\hline & & & \multirow{5}{*}{$93.83 b$} & & 93.69 & 1.891 & 3953151 & 2.859 & 6 & \multirow{5}{*}{40} & \\
\hline & & & & & 92.20 & 2.338 & 2043083 & 2.835 & 12 & & \\
\hline & & & & & 91.89 & 2.432 & 2124904 & 2.850 & 24 & & \\
\hline & & & & & 96.58 & 1.024 & 895346 & 2.779 & 48 & & \\
\hline & & & & & 94.80 & 1.560 & 1363063 & 2.859 & 96 & & \\
\hline & & & & & 84.91 & 4.525 & 3953151 & 2.966 & 6 & \multirow{5}{*}{50} & \\
\hline & & & & & 88.21 & 3.536 & 3089354 & 2.869 & 12 & & \\
\hline & & & $89.14 d$ & & 90.09 & 2.971 & 2595966 & 2.880 & 24 & & \\
\hline & & & & & 95.17 & 1.448 & 1265147 & 2.889 & 48 & & \\
\hline & & & & & 87.33 & 3.799 & 3319037 & 3.035 & 96 & & \\
\hline
\end{tabular}


Journal of Education and Science (ISSN 1812-125X), Vol: 30, No: 4, 2021 (161-176)

"المتوسطات ذات الاحرف غير المتثابهة في القطاع الواحد تثير الى وجود فروقات معنوية عند مستوى احتمال 5\%.

T. confusum بدول (3) نتائج التقييم الحيوي المختبري لمبيد Lambda-cyhalothrin بعريضه لارجات حرارة وفترات تعريض مختلفة ضد بالغات خنفساء الطحين المتشابهة

\begin{tabular}{|c|c|c|c|c|c|c|c|c|c|c|c|c|c|c|}
\hline \multirow{4}{*}{ تداخل الحرارة } & \multirow{4}{*}{ المتوسط العام للحرارة } & \multirow{4}{*}{ المتوسط العام للتركيز } & \multicolumn{10}{|c|}{ فترات التعريض/ساعة } & \multirow{4}{*}{ درجة الحرارة } & \multirow{4}{*}{$\begin{array}{c}\text { مادة فعالة } \\
\text { ppm }\end{array}$} \\
\hline & & & \multicolumn{10}{|c|}{ متوسط \% للقتل بعد/ ساعة } & & \\
\hline & & & \multicolumn{2}{|c|}{96} & \multicolumn{2}{|c|}{48} & \multicolumn{2}{|c|}{24} & \multicolumn{2}{|c|}{12} & \multicolumn{2}{|c|}{6} & & \\
\hline & & & 48 & 24 & 48 & 24 & 48 & 24 & 48 & 24 & 48 & 24 & & \\
\hline $0.567 \mathrm{a}$ & $1.25 \mathrm{a}$ & \multirow{3}{*}{$0.489 \mathrm{a}$} & 1 & 0.66 & 0.33 & 0 & 0.66 & 0.33 & 0.66 & 0 & 1.33 & 0.66 & 30 & \multirow{3}{*}{10} \\
\hline $0.267 \mathrm{a}$ & $1.083 \mathrm{a}$ & & 0.66 & 0.33 & 0.66 & 0 & 0 & 0 & 0.33 & 0 & 0.66 & 0 & 40 & \\
\hline $0.633 \mathrm{a}$ & $1.633 \mathrm{~b}$ & & 1.33 & 1.33 & 0.66 & 0 & 0 & 0 & 1.33 & 0.66 & 0.66 & 0.33 & 50 & \\
\hline $1.933 \mathrm{~b}$ & & \multirow{3}{*}{$2.156 b$} & 1.33 & 0.33 & 1 & 1 & 3 & 1.66 & 4.66 & 1.33 & 3.33 & 1.66 & 30 & \multirow{3}{*}{30} \\
\hline $1.9 \mathrm{~b}$ & & & 2 & 1.66 & 1.33 & 1.66 & 2.33 & 1.66 & 3 & 1 & 3 & 1.33 & 40 & \\
\hline $2.633 \mathrm{c}$ & & & 5.66 & 4.66 & 3.66 & 3.33 & 2.66 & 1.33 & 1.33 & 1.33 & 1.33 & 1 & 50 & \\
\hline & & & \multicolumn{2}{|c|}{$0.833 \mathrm{ab}$} & \multicolumn{2}{|c|}{$0.583 \mathrm{a}$} & \multicolumn{2}{|c|}{$1.417 \mathrm{~b}-\mathrm{e}$} & \multicolumn{2}{|c|}{$1.667 \mathrm{cde}$} & \multicolumn{2}{|c|}{$1.75 \mathrm{de}$} & 30 & \multirow{3}{*}{ تاخل التعريض x } \\
\hline & & & \multicolumn{2}{|c|}{$1.167 \mathrm{a}-\mathrm{d}$} & \multicolumn{2}{|c|}{$0.917 \mathrm{abc}$} & \multicolumn{2}{|c|}{$1 \mathrm{a}-\mathrm{d}$} & \multicolumn{2}{|c|}{$1 \mathrm{a}-\mathrm{d}$} & \multicolumn{2}{|c|}{$1.25 \mathrm{a}-\mathrm{e}$} & 40 & \\
\hline & & & \multicolumn{2}{|c|}{$3.25 \mathrm{f}$} & \multicolumn{2}{|c|}{$1.917 \mathrm{e}$} & \multicolumn{2}{|c|}{$1 \mathrm{a}-\mathrm{d}$} & \multicolumn{2}{|c|}{$1.167 \mathrm{a}-\mathrm{d}$} & \multicolumn{2}{|c|}{$0.833 \mathrm{ab}$} & 50 & \\
\hline & & & \multicolumn{2}{|c|}{$1.75 \mathrm{~b}$} & \multicolumn{2}{|c|}{$1.139 \mathrm{a}$} & \multicolumn{2}{|c|}{$1.306 \mathrm{a}$} & & & & & لفترة التعريض & المتوسط العا \\
\hline
\end{tabular}


وفي السياق ذاته ولتوضيح تأثير المعاملات الحرارية في خفض الكفاءة الحيوية للمبيد LCY جاءت النتائج الموضحة في الجدول (4) والخاصة بـ \% للتدور في فاعليه المبيد وبعد الرجوع الى نتائج التقييم الحيوي المبيد LCY قبل التعريض للدعاملات

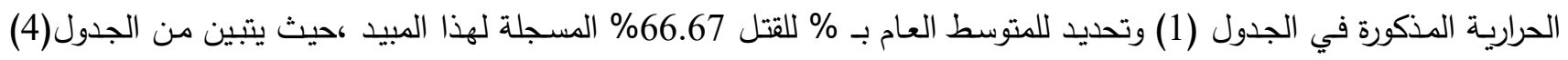

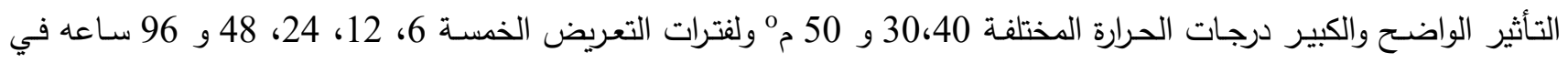
خفض الكفاءة الحيوية للمبيد بالتركيزين المستخدمين 10 و30 جزه بالمليون وان \% م للتدهور في فاعليه المبيد قد تراوحت ما بين


\% ولفترتي التعريض 24 و 48 ساعه 98.29 \% \%

وهذا ربما يؤشر كما سبق ذكره الي حساسيه هذا المبيد لدرجات الحرارة المستخدمة والتي كانت على ما يبدو مرتفعة في

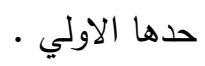

جدول (4) تأثير المعاملات الحرارية المختلفة في النسبة المئوية للتدهور في فاعلية مبيد Lambda-cyhalothrin ضد بالغات الحشره T.confusum

\begin{tabular}{|c|c|c|c|}
\hline \% للتدهور في فاعلية المبيد & المتوسط العام \% للقتل & & 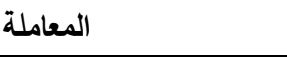 \\
\hline 99.26 & $0.489 \mathrm{a}$ & 10 & \multirow{2}{*}{ التركيز ppm / مادة فعالة } \\
\hline 96.76 & $2.156 \mathrm{a}$ & 30 & \\
\hline 98.12 & $1.25 \mathrm{a}$ & 30 & \multirow{3}{*}{ درجات الحرارة/مº } \\
\hline 98.37 & $1.083 \mathrm{a}$ & 40 & \\
\hline 97.55 & $1.633 \mathrm{~b}$ & 50 & \\
\hline 98.08 & $1.278 \mathrm{a}$ & 6 & \multirow{5}{*}{ فترات التعريض/ ساعة } \\
\hline 98.04 & $1.306 \mathrm{a}$ & 12 & \\
\hline 98.29 & $1.139 \mathrm{a}$ & 24 & \\
\hline 98.29 & $1.139 \mathrm{a}$ & 48 & \\
\hline 97.37 & $1.75 \mathrm{~b}$ & 96 & \\
\hline
\end{tabular}

"المتوسطات ذات الاحرف غير المتثابهة في القطاع الواحد تثير الى وجود فروقات مغنوية عند مستوى احتمال5\%.

\section{References:}

1. World Health Organization. (2013). International code of conduct on the distribution and use of pesticides: guidelines on data requirements for the registration of pesticides (No. WHO/HTM/NTD/WHOPES/2013.7). World Health Organization.

2. Tilman, D., Cassman, K. G., Matson, P. A., Naylor, R., \& Polasky, S. (2002). Agricultural sustainability and intensive production practices. Nature, 418(6898), 671-677.

3. Pimentel, D. , Environmental and economic cost of the application of pesticides primarily in the United States. (2005). Environ. Dev. Sustain. 7, 229-252.

4. Pimentel, D., \& Burgess, M. (2014). Environmental and economic costs of the application of pesticides primarily in the United States. In Integrated pest management (pp. 47-71). Springer, Dordrecht.

5. Leonard, R. A. (1990). Movement of pesticides into surface waters. Pesticides in the soil environment: processes, Impacts and Modeling, 2, 303-349.

6. Legrand, M. P., Costentin, E., \& Bruchet, A. (1991). Occurrence of 38 pesticides in various French surface and ground waters. Environ. Tech., 12(11), 985-996. 
7. Hernández, F., Morell, I., Beltran, J., \& López, F. J. (1993). Multi-residue procedure for the analysis of pesticides in groundwater application to samples from the comunidad valenciana, Spain. Chromatographia, 37(5), 303-312.

8. Matin, M. A., Malek, M. A., Amin, M. R., Rahman, S., Khatoon, J., Rahman, M., ... \& Mian, A. J. (1998). Organochlorine insecticide residues in surface and underground water from different regions of Bangladesh. Agri., Ecosys. Environ., 69(1), 11-15.

9. El-Kabbany, S., Rashed, M. M., \& Zayed, M. A. (2000). Monitoring of the pesticide levels in some water supplies and agricultural land, in El-Haram, Giza (ARE). J Hazardous Mat., 72(1), $11-21$.

10. Kashyap, R., Bhatnagar, V. K., \& Saiyed, H. N. (2002). Integrated pest management and residue levels of dichlorodiphenyltrichloroethane (DDT) and hexachlorocyclohexane $(\mathrm{HCH})$ in water samples from rural areas in Gujarat State, India. Archives of Environmental Health: An Int. J., 57(4), 337-339.

11. Shukla, G., Kumar, A., Bhanti, M., Joseph, P. E., \& Taneja, A. (2006). Organochlorine pesticide contamination of ground water in the city of Hyderabad. Environ. Int., 32(2), 244-247.

12. Maloschik, E., Ernst, A., Hegedűs, G., Darvas, B., \& Székács, A. (2007). Monitoring waterpolluting pesticides in Hungary. Microchem. J., 85(1), 88-97.

13. Spliid, N. H., \& Køppen, B. (1998). Occurrence of pesticides in Danish shallow ground water. Chemosphere, 37(7), 1307-1316.

14. Tuxen, N., Tüchsen, P. L., Rügge, K., Albrechtsen, H. J., \& Bjerg, P. L. (2000). Fate of seven pesticides in an aerobic aquifer studied in column experiments. Chemosphere, 41(9), 1485-1494.

15. Papadopoulou-Mourkidou, E., Karpouzas, D. G., Patsias, J., Kotopoulou, A., Milothridou, A., Kintzikoglou, K., \& Vlachou, P. (2004). The potential of pesticides to contaminate the groundwater resources of the Axios river basin in Macedonia, Northern Greece. Part I. Monitoring study in the north part of the basin. Sci. Total Environ., 321(1-3), 127-146.

16. Vara, S. (2012). Screening and evaluation of innate coagulants for water treatment: a sustainable approach. Int. J Energy Environ. Eng., 3(1), 1-11.

17. EPA (1988) Pesticide Fact Sheet Number 171: KARATE. U.S. Environmental Protection Agency, Office of Pesticide Programs,U.S. Government Printing Office: Washington, DC, p. 321.

18. Fetoui, H., Garoui, E. M., \& Zeghal, N. (2009). Lambda-cyhalothrin-induced biochemical and histopathological changes in the liver of rats: ameliorative effect of ascorbic acid. Exper. Toxic.

Pathol., 61(3), 189-196.

19. Ganeshwade, R. M. (2011). Biochemical changes induced by dimethoate in the liver of fresh water fish puntius ticto (HAM). In Biol. Forum-An Int. J. (Vol. 3, No. 2, pp. 65-68).

20. USEPA (US Environmental Protection Agency) (2008) Reregistration eligibility decision for cypermethrin (revised 1/14/08). OPP/2005/0293. US Environmental Protection Agency, Washington, DC.

21. EPA, E. (2009). Reregistration eligibility decision (RED) for Malathion. United States Environmental Protection Agency.

22. Shi, X., Gu, A., Ji, G., Li, Y., Di, J., Jin, J., ... \& Wang, X. (2011). Developmental toxicity of cypermethrin in embryo-larval stages of zebrafish. Chemosphere, 85(6), 1010-1016.

23. Housset, P., \& Dickmann, R. (2009). A promise fulfilled-pyrethroid development and the benefits for agriculture and human health. In Pyrethroid Scientific Forum 2009 (p. 135). 
24. Schleier III, J. J., \& Peterson, R. K. (2011). Pyrethrins and pyrethroid insecticides (Vol. 11, pp. 94-131). London: Royal Society of Chemistry.

25. Lubick, N. (2008). Pyrethroids are ubiquitous in California's urban streams.

26. Alonso, M. B., Feo, M. L., Corcellas, C., Vidal, L. G., Bertozzi, C. P., Marigo, J., ... \& Barceló, D. (2012). Pyrethroids: a new threat to marine mammals?. Environ. Int., 47, 99-106.

27. Aldehamee, M. H. M. (2015). Effect Of different Concentrations Of Pesticide Colti 5 (LambdaCyhalothrin) On Water Flea Daphnia pulex. J. Univ. Babylon, 23(2).

28. Gordon, C. J. (2005). Temperature and toxicology: an integrative, comparative, and environmental approach. CRC press.

29. Sparks, T. C., Pavloff, A. M., Rose, R. L., \& Clower, D. F. (1983). Temperature-toxicity relationships of pyrethroids on Heliothis virescens (F.)(Lepidoptera: Noctuidae) and Anthonomus grandis grandis Boheman (Coleoptera: Curculionidae). J Econ. Entomol., 76(2), 243-246.

30. Bloomfield, J. P., Williams, R. J., Gooddy, D. C., Cape, J. N., \& Guha, P. M. (2006). Impacts of climate change on the fate and behaviour of pesticides in surface and groundwater - a UK perspective. Sci. Total Environ., 369(1-3), 163-177.

31. European Food Safety Authority (EFSA). (2008). Opinion on a request from EFSA related to the default Q10 value used to describe the temperature effect on transformation rates of pesticides in soil-Scientific Opinion of the Panel on Plant Protection Products and their Residues (PPR Panel). EFSA J., 6(1), 622.

32. Reuhs B.L., Rounds M.A. (2010) High-Performance Liquid Chromatography. In: Food Analysis. Food Analysis. Springer, Boston, MA. https://doi.org/10.1007/978-1-4419-1478-1_28.

33. Reuhs, B. L. (2017). High-performance liquid chromatography. In Food analysis (pp. 213-226). Springer, Cham.

34. Lazić, S., Šunjka, D., Grahovac, N., Guzsvány, V., Bagi, F., \& Budakov, D. (2012). Application of liquid chromatography with diode-array detector for determination of acetamiprid and 6chloronicotinic acid residues in sweet cherry samples. Pesticidi i Fitomedicina, 27(4), 321-329.

35. Chauhan, S. S., Agrawal, S. A. N. J. E. E. V., \& Srivastava, A. N. J. A. N. A. (2013). Effect of imidacloprid insecticide residue on biochemical parameters in potatoes and its estimation by HPLC. Asian J. Pharm. Clin. Res., 6(3), 114-117.

36. Kithure, J. G., Murungi, J. I., Wanjau, R. N., \& Thoruwa, C. L. (2014). Analysis of deltamethrin residue amounts using hplc in some vegetables consumed in a rural areas. Case study of Makuyu in Kenya.

37. Sharma, S., Mandal, K., \& Singh, B. (2014). Sensitive methodology for simultaneous determination of residues of imidacloprid and its metabolites in sugarcane leaves and soil. $J$. AOAC Int., 97(4), 1183-1188.

38. Hamid, a., yaqub, g., ayub, m., \& naeem, m. (2020). Determination of malathion, chlorpyrifos, $\lambda$ cyhalothrin and arsenic in rice. Food Sci. Technol., (ahead).

الملاح ، نزار مصطفى(2014). الاساسيات في علم سموم مبيدات الحشرات. العُلا للطباعة والنشر -الموصل ، 481 صفحة. .

40. Sabbour, M. M. (2015). Efficacy of some nano-Imidacloprid against red flour beetle Tribolium confusum (Coleoptera Tenebrionidae) under laboratory and stored conditions. Advances Bioch. Biotechnol., 1(1), 1-13. 
41. Abbot, W. B.(1925). A method of computing the effectiveness of an insecticide. J. Econ. Entomol. 18: 265-267.

42. Shaaban, Awwad and Nizar Mustafa Al-Mallah (1993). pesticides. Directorate of Dar Al-Kutub for Printing and Publishing, University of Mosul, 520. (In Arabic).

43. Al-Mallah, Nizar Mustafa and Abdul Razzaq Younis Al-Jubouri. (2013). Scientific applications in pesticides. Dar Al-Yazori Publishing, Amman, Jordan. 537 p. (In Arabic).

44. Hammad, A. M., Yasein, B. E. H., Abd Elaziz, S. A., Abdelbagi, A. O., \& Laing, M. D. (2015). Detection of insecticide residues on tomato fruits grown in greenhouses in Khartoum State. Univ. Khartoum J. Agri. Sci., 23(1).

45. AlJuboori,I.F.A.(2012).Lipid production from some local algae at different cultivation conditions University of Baghdad in partial fulfillment of the requirements for the Degree of Master of Science in Biology/Botany.

46. Antar, Salem Hammadi (2010). Statistical Analysis in Scientific Research and SAS Program. University of Mosul. College of Agriculture and Forestry. House of Books and Publishing, 192 pages. (In Arabic).

47. Jankov, D., Inđić, D., Kljajić, P., Almaši, R., Andrić, G., Vuković, S., \& Grahovac, M. (2012). Initial and residual efficacy of insecticides on different surfaces against rice weevil Sitophilus oryzae (L.). J. Pest Sci., 86(2), 211-216.

48. Grávalos, C., Fernández, E., Belando, A., Moreno, I., Ros, C., \& Bielza, P. (2015). Crossresistance and baseline susceptibility of Mediterranean strains of Bemisia tabaci to cyantraniliprole. Pest Management Sci., 71(7), 1030-1036.

49. Khalequzzaman, M., \& Nahar, J. (2001). Toxicity of nine insecticides to adult Tribolium castaneum (Herbst). Online J. Biol. Sci., 1(11), 1043-1045.

50. Starner, K., Kuivila, K. M., Jennings, B., \& Moon, G. E. (1999). Degradation rates of six pesticides in water from the Sacramento River, California. US Geological Survey Toxic Substances Hydrology Program Water Resource Investigation Rep, 89-99.

51. Castillo, M. D. P., \& Torstensson, L. (2007). Effect of biobed composition, moisture, and temperature on the degradation of pesticides. J. Agri. Food Chem., 55(14), 5725-5733.

52. Mohammed, E. A., \& Mousa, S. A. (2018). Degradation of Dazomet by thermal Fenton and photo-Fenton processes under UV and sun lights at different temperatures. Bag. Sci. J., 15(2), 158-168.

53. Kumar, N., \& Gupta, S. (2020). Persistence and degradation of cyantraniliprole in soil under the influence of varying light sources, temperatures, moisture regimes and carbon dioxide levels. $J$. Environ. Sci. Health, Part B, 55(12), 1032-1040.

54. Fernández-Alberti, S., Rubilar, O., Tortella, G. R., \& Diez, M. C. (2012). Chlorpyrifos degradation in a Biomix: Effect of pre-incubation and water holding capacity. J Soil Sci. Plant Nut., 12(4), 785-799.

55. Scott, J. G. (1995). Effects of temperature on insecticide toxicity. Reviews in pesticide toxicology.

56. Thompson, H. M. (2001). Assessing the exposure and toxicity of pesticides to bumblebees (Bombus sp.). Apidologie, 32(4), 305-321.

57. Raj Boina, D., Onagbola, E. O., Salyani, M., \& Stelinski, L. L. (2009). Influence of posttreatment temperature on the toxicity of insecticides against Diaphorina citri (Hemiptera: Psyllidae). J. Economic Entomol., 102(2), 685-691. 
58. Srigiriraju, L., Semtner, P. J., \& Bloomquist, J. R. (2010). Influence of post-exposure temperature on the toxicity of insecticides to the tobacco-adapted form of the green peach aphid (Hemiptera: Aphididae). J. Entomol. Sci., 45(2), 178-187.

59. Ma, Y. H., Gao, Z. L., Dang, Z. H., Li, Y. F., \& Pan, W. L. (2012). Effect of temperature on the toxicity of several insecticides to Apolygus lucorum (Meyer-Dür). J. Pesticide Sci., 12030902271203090227.

60. Samuel, T., \& Pillai, M. K. K. (1988). The effect of temperature and solar radiations on volatilisation, mineralisation and degradation of [14C]-DDT in soil. Environ. Pollution, 57(1), 63-77.

61. Li, H., Feng, T., Liang, P., Shi, X., Gao, X., \& Jiang, H. (2006). Effect of temperature on toxicity of pyrethroids and endosulfan, activity of mitochondrial $\mathrm{Na}+-\mathrm{K}+-\mathrm{ATPase}$ and $\mathrm{Ca} 2+-\mathrm{Mg} 2+-$ ATPase in Chilo suppressalis (Walker)(Lepidoptera: Pyralidae). Pesticide Biochem. Physiol., 86(3), 151-156.

62. Satpute, N. S., Deshmukh, S. D., Rao, N. G. V., Tikar, S. N., Moharil, M. P., \& Nimbalkar, S. A. (2014). Temperature-dependent variation in toxicity of insecticides against Earias $\mathrm{V}$ itella (Lepidoptera: Noctuidae). J. Econ. Entomol., 100(2), 357-360.

63. Mao, K., Jin, R., Li, W., Ren, Z., Qin, X., He, S., ... \& Wan, H. (2019). The influence of temperature on the toxicity of insecticides to Nilaparvata lugens (Stål). Pesticide Biochem. Physiol., 156, 80-86.

64. Narahashi, T., Carter, D. B., Frey, J., Ginsburg, K., Nagata, K., Roy, M. L., ... \& Tatebayashi, H. (1995). Sodium channels and GABAA receptor-channel complex as targets of environmental toxicants. Toxicol. Let., 82, 239-245. 